\title{
MYCN amplicon junctions as tumor-specific targets for minimal residual disease detection in neuroblastoma
}

\author{
HANNA KRYH $^{1}$, JONAS ABRAHAMSSON ${ }^{2}$, ELSA JEGERÅ ${ }^{1}$, ROSE-MARIE SJÖBERG ${ }^{1}$, \\ IRENE DEVENNEY $^{3}$, PER KOGNER ${ }^{4}$ and TOMMY MARTINSSON ${ }^{1}$
}

\author{
${ }^{1}$ Department of Clinical Genetics, Institute of Biomedicine, The Sahlgrenska Academy at the University of Gothenburg, \\ SE-41345 Gothenburg; ${ }^{2}$ Institute of Clinical Sciences, The Sahlgrenska Academy at the University of Gothenburg, \\ SE-41685 Gothenburg; ${ }^{3}$ Institute of Clinical and Experimental Medicine, University of Linköping, SE-58183 \\ Linköping; ${ }^{4}$ Childhood Cancer Research Unit, Karolinska Institutet, SE-17176 Stockholm, Sweden
}

Received April 14, 2011; Accepted June 2, 2011

DOI: 10.3892/ijo.2011.1120

\begin{abstract}
The $M Y C N$ gene is frequently amplified in unfavorable neuroblastoma tumors. Therefore, this study aimed at characterizing the novel junctions connecting the amplified DNA segments (amplicons) and obtaining tumor-specific PCR fragments for use in detecting minimal residual disease (MRD). High-density SNP arrays were used to map the end-points of the MYCN amplicons in a subset of neuroblastoma tumors. Primers were designed to give rise to a tumor-specific PCR product and were examined for MRD in the blood and bone marrow using quantitative PCR. Tumor-specific junction fragments were detected in all cases, confirming a head-to-tail tandem orientation of the amplicons and revealing microhomology at the amplicon junctions, thus suggesting a rolling circle caused by microhomology-mediated break-induced replication (MMBIR) as a possible mechanism initiating the MYCN amplification. We also evaluated the use of these junctions as tumor-specific targets for detecting MRD and observed that tumor DNA could be readily detected and quantified in either blood or bone marrow at a sensitivity of $1 / 10^{6}$ tumor/control DNA. This study provides new information on the mechanisms of oncogene amplification and envisages means of rapidly obtaining highly sensitive PCR-based tools for tumor/ patient-specific monitoring of treatment response and the early detection of relapse in patients with neuroblastoma.
\end{abstract}

\section{Introduction}

Neuroblastoma (NB) is one of the most common solid tumors of childhood and accounts for about $15 \%$ of all childhood

Correspondence to: Dr Tommy Martinsson, Department of Clinical Genetics, Institute of Biomedicine, The Sahlgrenska Academy at the University of Gothenburg, SE-41345 Gothenburg, Sweden

E-mail: tommy.martinsson@gu.se

Key words: MYCN amplification, microhomology, microhomologymediated break-induced replication, minimal residual disease, neuroblastoma cancer deaths (1). A subgroup of aggressive NB is characterized by high-grade amplification of the $M Y C N$ proto-oncogene and of a substantial region flanking it in both directions $(2,3)$. The amplified region is variable in size, and although a core region that includes the $M Y C N$ gene is always present, other genes in the close vicinity, such as $D D X 1$, are frequently co-amplified (4). The original location of $M Y C N$ is $2 \mathrm{p} 24$, but on chromosomal slides from tumor preparations the amplified fragments are typically seen as either double minute chromosomes (DMs) or as homogeneously staining regions (HSRs) located on chromosomal sites other than $2 p(1,5,6)$.

Several different models attempting to explain the mechanisms that underlie oncogene amplifications have been proposed, most of which are based on studies of drug-induced amplifications $(6,7)$. These models use either re-replication or recombination to explain a variety of amplifications, differing both with respect to the location and orientation of the amplicon units, as well as to the amount of homology found at the junctions. Moreover, in some cases the native locus has been found to be excised, whereas in other cases the native locus remains intact. This heterogeneity among amplicons suggests that probably more than one mechanism is responsible for causing amplification. The MYCN amplicon in NB has previously been characterized as a direct head-to-tail repeat (8), situated outside the original locus (6). However, whether the native locus stays intact or not remains somewhat contradictory $(6,9)$. None of these earlier publications studied the junction region at the DNA sequence level.

With the development of sensitive quantification of minimal residual disease (MRD), early response to treatment has emerged as the most important prognostic factor in childhood acute leukemia and is used for treatment stratification. Although the $M Y C N$ amplification has been studied in NB for more than 20 years (10), the prognosis for this group of patients remains poor. It is well established that the extent of disease after induction therapy, as evaluated by MIBG scintigraphy, imaging, bone marrow cytology and urinary catecholamine, has prognostic significance. However, at this late time-point, salvage treatment for those with poor response is often ineffective. Since the majority of children with $M Y C N$ 
amplification have bone marrow metastases it is possible that MRD measurement with high sensitivity could lead to early detection of resistant tumors, thus allowing for treatment modification. A recent study, using a combination of five molecular markers showed that those with negative MRD at mid-induction or after induction had a much better outcome than those with measurable disease (11).

Several versions of quantitative PCR can be used for MRD detection; traces of tumor can be detected either at the transcript level using cDNA as a template, or at the genomic level using DNA to measure copy-number changes in regions known to be amplified or rearranged. Since no recurrent fusion genes occur in NB, MRD using transcripts requires a well-defined set of target genes that can discriminate between tumor and blood mRNA. In NB, studies disagree as to what target genes are the most suitable and recent papers recommend using a panel of multiple genes to improve the sensitivity (11-13).

In leukemia and lymphoma, MRD detection with PCR analyses directed against tumor-specific junctions is routinely used in many treatment protocols (14). It has not been possible to adopt this strategy in MRD studies of NB tumors because of the lack of good junction targets. A quick, robust way of generating highly sensitive patient/tumor-specific PCR primers for detecting MRD would help in the monitoring and treatment of this group of patients. While this manuscript was in preparation, McBride et al (15) and Leary et al (16) have shown that next generation sequencing can be used on solid tumors such as breast cancer, colorectal cancer and osteosarcoma to identify tumor/patient specific junctions suitable for MRD detection. Although promising, the high costs associated with this approach currently prohibit a widespread use in a clinical setting.

In this article we show that SNP-arrays, commonly used in neuroblastoma diagnostics, is a cost-efficient way to identify tumor specific junctions that are suitable targets for MRD detection. Here we have focused on the MYCN amplification often present in aggressive neuroblastoma tumors and have generated primers for each patient, detecting a tumor- and patient-specific PCR fragment across the amplicon-junction. We also test the use of these junctions as tumor-specific targets for detecting MRD and show that tumor DNA can be readily detected and quantified in either blood or bone marrow from patients with $M Y C N$ amplification. Furthermore, we present a detailed analysis at the sequence level of the $M Y C N$ amplicon junctions and discuss possible mechanisms by which these amplicons are generated.

\section{Materials and methods}

Samples and breakpoint mapping. Fourteen primary NB tumors and 3 NB cell lines, previously analyzed using high-density SNP arrays (Genechip 250K Nsp, Affymetrix Inc., Santa Clara, CA) (2), were chosen because they presented a single well-defined peak of amplification surrounding the $M Y C N$ locus. The array data was thoroughly re-examined using either Copy Number Analyzer for GeneChip (CNAG 3.0; Genome Laboratory, Tokyo University, http://www.genome. umin.jp) or Genotyping Consol (Affymetrix) to determine the breakpoint intervals as precisely as possible. In a few cases an additional array (Genechip 250K Sty or SNP 6.0, Affymetrix) was used to improve the resolution. The experimental procedure has previously been described (2). Two samples (NBL12 and NBL13) were found to consist of more complex amplicons than revealed by the first analysis and were therefore excluded from the study. Four of the tumors were selected for further analyses due to their small breakpoint intervals (NBL05, NBL10) or because the patients were currently in treatment (NBL08, NBL11). For the two clinical cases, blood samples were also retrieved at different time-points during treatment. DNA was prepared from whole blood using the DNeasy Blood and Tissue kit (Qiagen, Hilden, Germany). Brief information about the clinical course of these patients is presented in Fig. 4C. The research has been carried out in accordance with the Declaration of Helsinki (2000) of the World Medical Association. Ethical permission was granted by the local ethics committee and informed consent was obtained from the parents of the patients.

PCR amplification and DNA sequencing. The genomic positions of the breakpoint regions were obtained from the UCSC genome browser May 2004 (NCBI35/hg17) and March 2006 (NCBI36.1/hg18) (www.genome.ucsc.edu) using information from the array data on the last non-amplified and first amplified SNP probe flanking the amplicon. Primers were designed using Primer 3 (http://frodo.wi.mit.edu/primer3), and purchased from Invitrogen (Carlsbad, CA). In a few cases where breakpoint regions were extensive, universal tags were added to the primers, making multiplex PCR reactions possible. Primers for detecting amplicon junctions were designed across the entire breakpoint regions, $\sim 1$ primer/ $\mathrm{kb}$, facing outward towards the suspected break. Primers were also designed facing inward to be able to detect a deletion in the same region as the amplification. These primers, however, were designed secondary to the analysis of the amplicon-PCR, and were therefore designed specifically for the region detected in the first PCR reaction. In NBL08, an additional set of deletion primers was also designed for the upstream breakpoint, $\sim 1$ primer $/ \mathrm{kb}$. Primer sequences are available upon request.

PCR reactions were performed in a total volume of $25 \mu \mathrm{l}$ containing $50 \mathrm{ng}$ of genomic DNA, $0.8 \mu \mathrm{M}$ of each primer, $1 \mathrm{X}$ buffer from the supplier (containing $1.5 \mathrm{mM} \mathrm{MgCl}$ ), $200 \mu \mathrm{M}$ of each dNTP and $0.65 \mathrm{U}$ HotstarTaq polymerase (Qiagen). Touchdown PCR was used for amplification with an initial denaturation at $95^{\circ} \mathrm{C}(10 \mathrm{~min})$ followed by 35 cycles of denaturation $95^{\circ} \mathrm{C}(30 \mathrm{sec})$, annealing $67-57^{\circ} \mathrm{C}$ (30 sec) $(20$ cycles with a decrease of $0.5^{\circ} \mathrm{C} /$ cycle, and 15 cycles at $57^{\circ} \mathrm{C}$ ) and extension $72^{\circ} \mathrm{C}$ (3 min). Finally, an extension at $72^{\circ} \mathrm{C}$ for $10 \mathrm{~min}$ was performed. The above settings were used as a starting point for the PCR reactions. However, annealing temperatures were changed in a few cases to improve the specificity of the product prior to DNA sequencing. In one case, gel extraction of the PCR product using the QIAEX II Gel extraction kit (Qiagen) was also performed. Multiplex PCR was performed with up to 12 primers in the same reaction using the same conditions as described above. In all PCR reactions, control DNA from a healthy blood donor was also run in parallel with the tumor DNA to ensure the tumor specificity of the fragments.

For each sample, the most suitably sized PCR product was purified using Agencourt AMPure magnetic beads (Beckman Coulter, Brea, CA) and eluted in $\mathrm{dH}_{2} \mathrm{O}$. The sequencing PCR was 

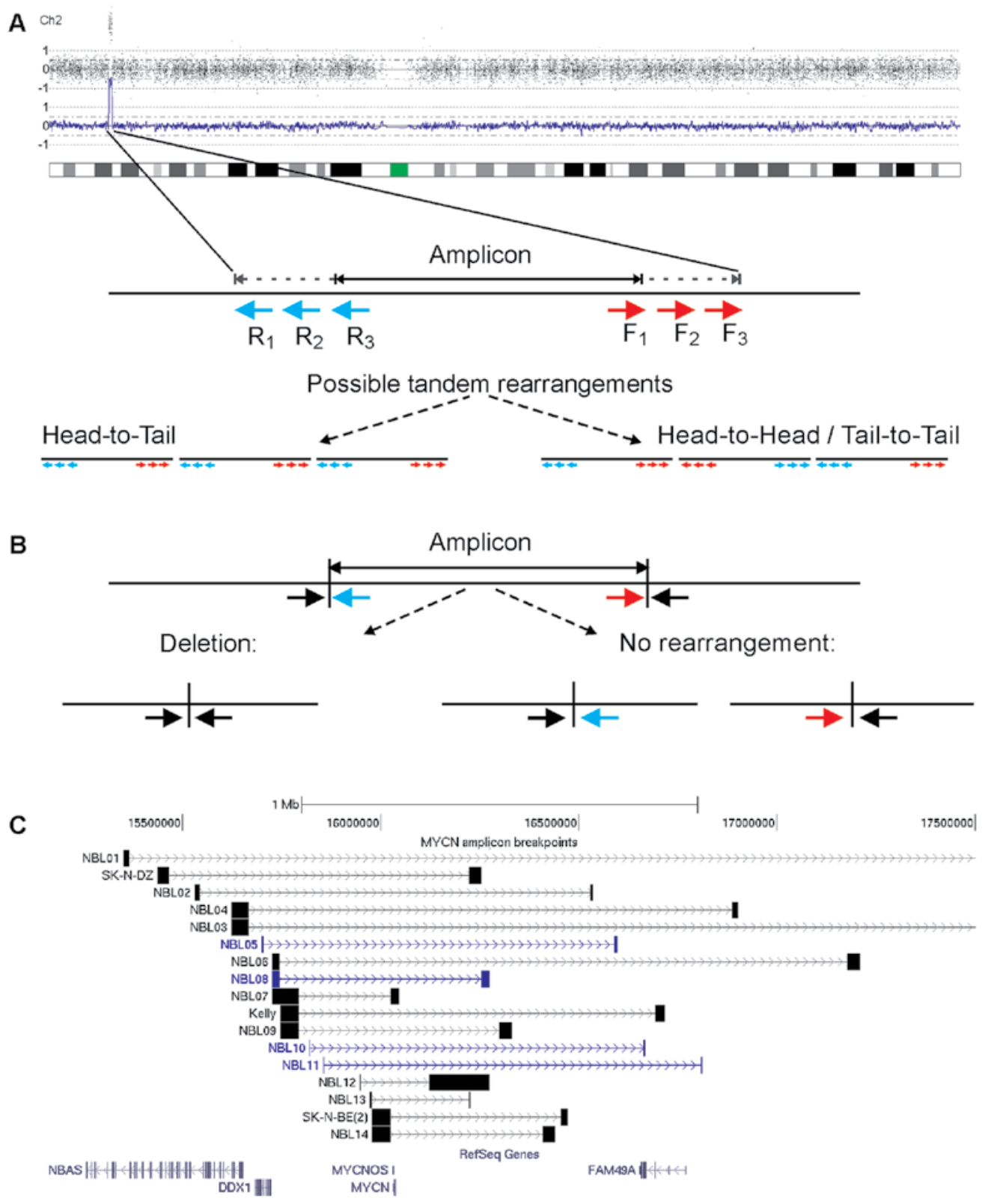

Figure 1. Primer design and layout. (A) High density SNP-array data were used to define the breakpoints as precisely as possible for each tumor. The breakpoint region was then saturated with primers facing outward, enabling detection of the amplicon junctions. (B) Primers were also designed facing inward to be able to detect a deletion in the same region as the amplicon. These primers were also used to confirm the functionality of the amplicon primers. (C) Genomic positions of the amplicons according to UCSC 2006 (hg18), with the four samples (NBL05, NBL08, NBL10, NBL11) chosen for detailed analysis highlighted in blue. The proximal breakpoints of NBL01 and NBL03 (located at 17.8 and 19.9 Mb, respectively) are not shown. Vertical bars represent the breakpoint intervals as defined from the SNP-array. RefSeq genes in the area are also shown for comparison.

then performed in $10 \mu \mathrm{l}$ reactions containing 1.6 pmol primer, $6 \mu \mathrm{l}$ of $1: 3$ diluted PCR product, $0.25 \mu \mathrm{l}$ BDT v3.1 and $1 \mathrm{X}$ BDT buffer (Applied Biosystems, Foster City, CA, USA). Cycling conditions were $96^{\circ} \mathrm{C}(1 \mathrm{~min})$, followed by 40 cycles of $96^{\circ} \mathrm{C}$ $(10 \mathrm{sec}), 50^{\circ} \mathrm{C}(5 \mathrm{sec})$ and $60^{\circ} \mathrm{C}$ for $2 \mathrm{~min}$ and $30 \mathrm{sec}$. Sequencing products were then purified using Agencourt CleanSeq magnetic beads (Beckman Coulter), re-suspended in $10 \mu \mathrm{l}$ of High Dye formamide (Applied Biosystems) and analyzed using an ABI3730 DNA analyzer (Applied Biosystems). All fragments were bidirectionally sequenced, using either the same primers that were used for PCR amplification or primers directed against the universal tag. Data was analyzed using Sequence Analysis (Applied Biosystems), SeqScape (Applied Biosystems), BLAT at the UCSC genome browser (www.genome.ucsc.edu) version May 2004 (NCBI35/hg17) and March 2006 (NCBI36.1/hg18) and BLAST at NCBI (www.ncbi.nlm.nih.gov).

MRD detection. For the two clinical cases (NBL08, NBL11), DNA from a series of blood or bone marrow samples was used as a template in both a semi-quantitative and a quantitative PCR reaction. The semi-quantitative PCR reactions were performed as described above, using the primers previously shown to give rise to a patient/tumor-specific junction PCR fragment. DNA (50 ng) was used as a template in all cases except B2 and BM for NBL11, where the low quality and quantity of DNA made quantifications dubious. A $20 \mu$ l sample of 
A

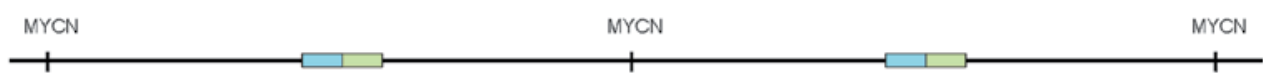

B NBL05/NBL10 / NBL11

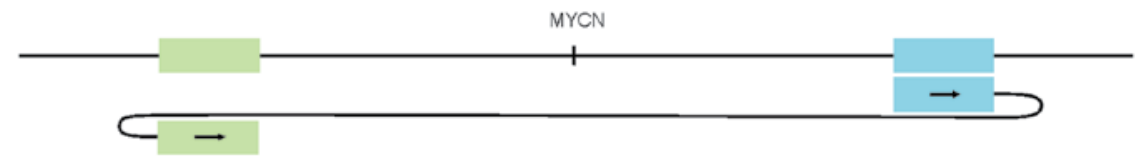

C NBL08

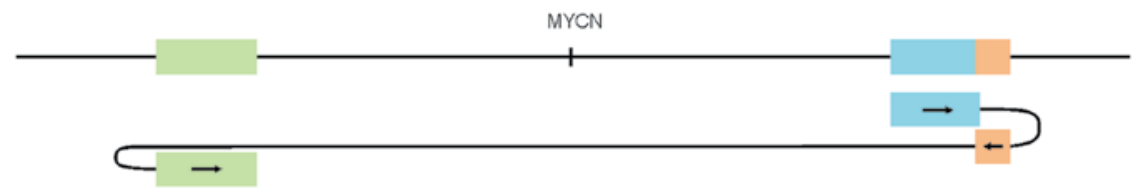

D NBL05

Rearrangement AACCCTAAGCATCATATAAAATAGAGGTGCATTTTTAAATATATGTAAAATGAATAAAATAATATTTTTTCCCATAGAAAGTGATGAAC

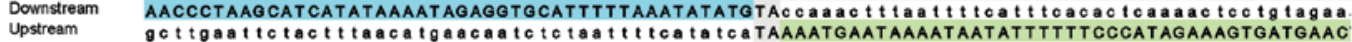

NBL08

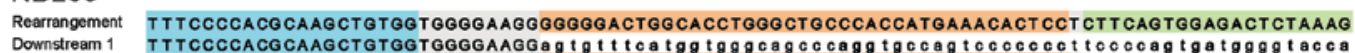

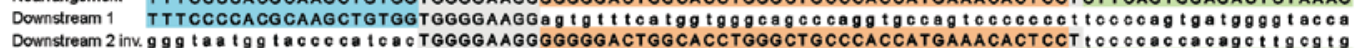

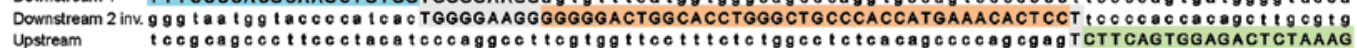

NBL10

Rearrangement GGATGTAGGCTAGTCTGAAGGGAAAAAGCCACCGGGAGGGAATTAGTGATACTTTGGGCCGTGAGCCTGGAGAAGCGTTCTGGTCTTAG

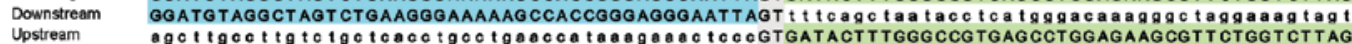

NBL11

Rearrangement ATAATCAAAATQAGATTOCTTTCATOTGCAAAAGTGAGCTOAAGACCCTAATAT TTCCTTCTOGCCTCATCTCCCACTATCAOTQAATO Downstream a a tcc caact tccaggo t tgaac t t taagotco tcaccatc togCCCTAATATTTCCTTCTGGCCTCATCTCCCACTATCAGTGAATG

Figure 2. Amplicon junctions for four cases of $M Y C N$ amplification. (A) Schematic picture of the structure of the amplicons. Multiple amplicon units are connected to each other in a head-to-tail arrangement. (B) Schematic picture of the reference sequence and the rearrangement in the tumors NBL05, NBL10 and NBL11. (C) Schematic picture of the reference sequence and the rearrangement in the tumor NBL08. (D) The junction sequence aligned to the reference sequence at the upstream and downstream breakpoints of the respective amplicons. The sequenced junction fragments all reveal a few bases of microhomology at the junctions. The sequenced junction fragment of NBL08 also reveals a small inversion in the vicinity of the actual head to tail junction.

unknown concentration was used instead. A serial dilution of tumor DNA from the same patient, mixed with control DNA from a healthy blood donor, was used as a standard and was run in parallel with the blood samples in the PCR. Fragments were then visualized on agarose gels and compared with the standard as a rough estimate of tumor DNA infiltration in the blood. The resulting PCR products were also sequenced as described above to ensure that the correct junction fragment was detected.

For QPCR analysis, additional primers were designed, yielding small PCR fragments of $\sim 50-150$ bp spanning the amplicon junction (primer sequences are available upon request). QPCR reactions were performed in a volume of $10 \mu \mathrm{l}$, containing $300 \mathrm{nM}$ of each primer and $1 \mathrm{X}$ Power SYBR ${ }^{\circledR}$ Green buffer (Applied Biosystems) using a 7900HT sequence detection system (Applied Biosystems). Cycling conditions were $95^{\circ} \mathrm{C}(10 \mathrm{~min})$ followed by 40 cycles of $95^{\circ} \mathrm{C}(15 \mathrm{sec})$ and $60^{\circ} \mathrm{C}(1 \mathrm{~min})$. A dissociation curve was also included to ensure the specificity of the amplified fragments. DNA, $25 \mathrm{ng}$, was used as a template in all cases, except B2 which was excluded, and BM from patient NBL11, where $1 \mu \mathrm{l}$ of unknown concentration was used instead. Samples were run in triplicates, and a primer set targeting a region at $20 \mathrm{q} 13.2$ containing 2 copies in these tumors, as determined from the array analysis, was used as an endogenous control. Results are given as amplicon junction copies/cell $\left\{2^{*} 2^{\wedge}[\mathrm{Ct}(\mathrm{Amp})-\mathrm{Ct}(\mathrm{Ctrl})]\right\}$ where 'Amp' is the amplicon junction and 'Ctrl' is the control region at 20q13.2.

\section{Results}

Detailed analysis reveals microhomology at the amplicon junctions. SNP-array data from a set of 14 NB tumors and 3 cell lines, all presenting with high grade amplification of the MYCN gene, was analyzed with respect to the localization of the breakpoints delimiting these amplicons. The positions of the breakpoints were found to be scattered around $M Y C N$ in a non-recurrent fashion, with only a few breakpoint regions found in more than one tumor (Fig. 1). The three most distal breakpoints were also found to map to the recently described fragile site FRA2Ctel at 2p24.3 (17) although the majority of breakpoints were located slightly proximal to this region. A subset of 4 tumors was then chosen for further analysis, and primers were designed to generate PCR products spanning the amplicon junctions. 
A NBL08

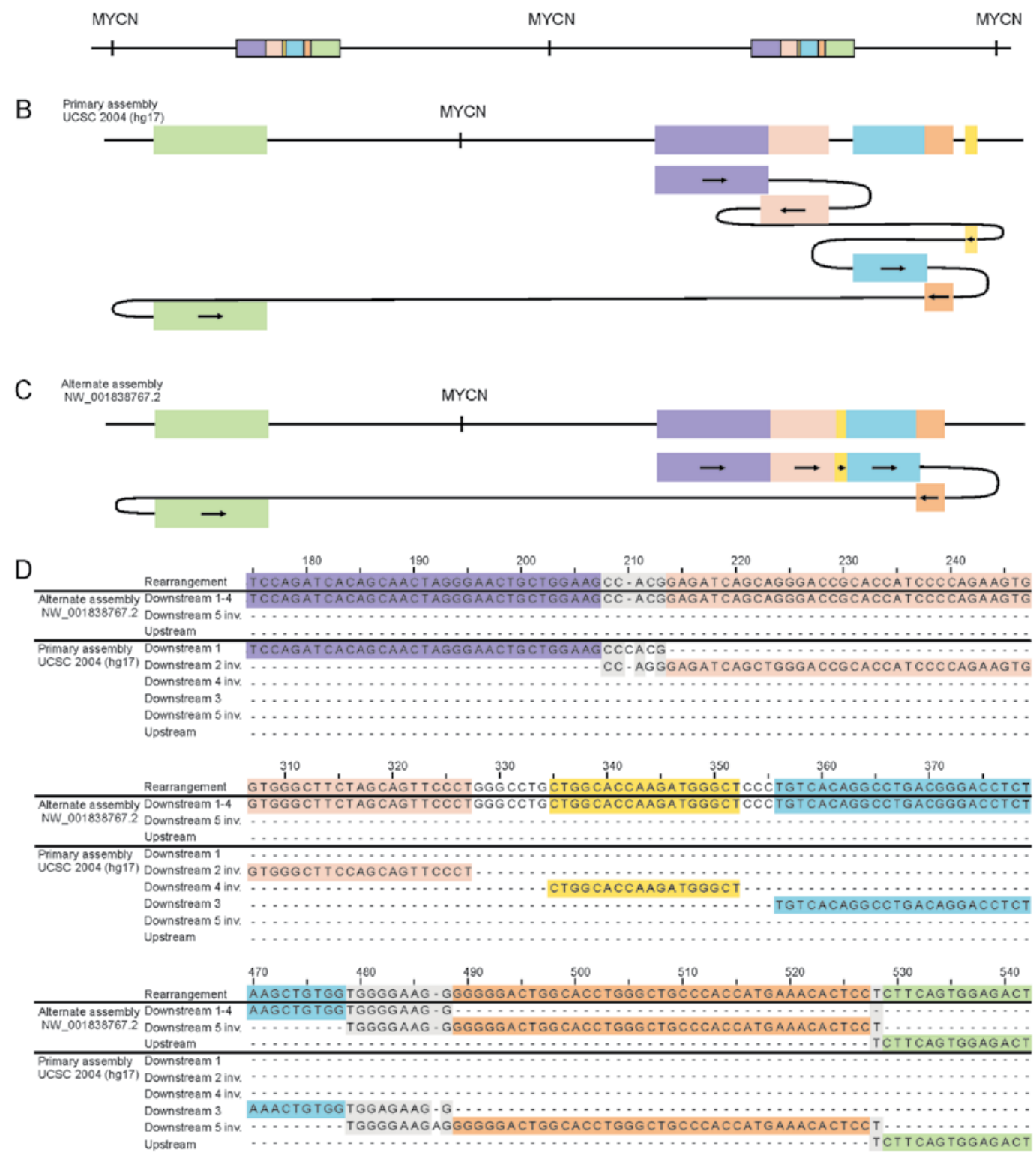

Figure 3. The amplicon junction in NBL08: comparison between different genomic assemblies. (A) Schematic picture of the amplicon structure. Multiple amplicon units are connected to each other in a head-to-tail arrangement. (B) Schematic picture of the reference sequence and the rearrangement as proposed by UCSC 2004 (NCBI35/hg17). (C) Schematic picture of the reference sequence and the rearrangement as proposed by the alternate assembly based on HuRef (NW_001838767.2). (D) Alignment of the rearranged sequence to either UCSC 2004 (NCBI35/hg17) or the alternate assembly (NW_001838767.2). The alternate assembly provides the best match, leaving only one inversion and the actual head-to-tail junction to be explained by the amplification event. The alignment has been cropped to include only stretches of sequence where rearrangement has occurred or where the assemblies differ from each other.

Tumor-specific PCR fragments were successfully obtained and sequenced for all of the four samples analyzed (Fig. 2). The resulting sequences were then mapped to the reference genome using BLAT or BLAST resources at the UCSC and NCBI, respectively. All of the junction fragments mapped to separate positions on either side of $M Y C N$, corresponding to their breakpoint regions as estimated from the array results. Detailed analysis of the junction sequences revealed very simple overlaps at the junctions, with only a small microhomology of 1-4 bases connecting the amplicon units head to tail. For one of the junction fragments (NBL08), the initial BLAT analysis (UCSC May 2004 and March 2006) displayed a more complex pattern, with five rearrangements occurring in the vicinity of the junction. These internal rearrangements showed either a microhomology of 1-9 bases or a gap of 3-6 bases that could not be mapped. BLAST analysis of the same sequence, however, resulted in another, more suitable hit (NW_001838767.2, alternate assembly based on HuRef) that explained most of the internal rearrangements (Fig. 3), leaving only one small inversion and the actual head-to-tail junction to be explained by the MYCN amplification (Figs. 2D and 3D).

Primers designed to detect a deletion in the same region as the amplicon did not result in PCR products in any of the tumors studied. This was also the case for NBL08, where a deletion, extending a further $1.4 \mathrm{Mb}$ upstream of the amplicon site was inferred from the array data. Although an additional set of primers was designed for this distal breakpoint, no deletion could be detected, probably reflecting either a more 
complex deletion or a translocation event. In contrast, the deletion primers, used in combination with their corresponding amplicon primers, gave rise to distinct PCR products as expected in both the tumor and healthy control, thus confirming that the primers were functional and annealed to the correct position. The resulting PCR products were also confirmed by sequencing and were found to map to the expected position on the reference assembly (UCSC 2004 and 2006) in all cases except the downstream breakpoint of NBL08, where the alternate assembly described above provided a better match. To verify this finding, three additional control samples were also sequenced, all confirming the alternate assembly as a better match for this region. Therefore, this may reflect an error in the GRCh37 primary assembly (NT_015926.15).

Tumor DNA can be detected in blood and bone marrow. The primer combinations that detected a tumor-specific amplicon fragment were also tested for MRD detection using both semiquantitative and quantitative PCR assays (Fig. 4). The two tumors that were analyzed in this part of the study (NBL08 and NBL11) were derived from patients currently undergoing treatment, and blood and bone marrow samples from these patients were taken during regular check-ups at different points in time after diagnosis. To find out whether our method was sensitive enough to detect small amounts of tumor DNA in blood samples, a semi-quantitative PCR reaction was set up using a serial dilution of tumor/control DNA as a standard (Fig. 4A). This simple gel-based assay was found to be capable of detecting the tumor-specific fragment down to a dilution of $1 / 10^{5}$ and $1 / 10^{6}$ tumor/control DNA in NBL08 and NBL11, respectively. Real-time QPCR analysis was found to have approximately the same sensitivity (Fig. 4B).

In both analyses, NBL08 was found to contain tumor DNA in the first three blood samples, with a tendency to decrease over time. A second sample from the surgically removed primary tumor was also analyzed and found to contain the same amplicon junction. The fourth blood sample was found to be negative. In the case of NBL11, the semi-quantitative assay was positive for the first blood sample B1 and for the following blood and bone marrow sample (B2 and BM). In the last two blood samples (B3 and B4) however, tumor- specific fragments could not be detected. The QPCR assay gave comparable results for all samples except $\mathrm{B} 2$ which was excluded due to lack of material.

\section{Discussion}

Microhomology at the amplicon junctions suggests MMBIR as a mechanism for amplicon initiation. All of the junctions studied revealed a fairly simple rearrangement with only small microhomologies of 1-9 bases at the junctions. In three of the tumors a simple junction was found, connecting the amplicon units head-to-tail, whereas the fourth case consisted of a slightly more complex rearrangement with a small inversion in the vicinity of the actual head-to-tail junction. This type of rearrangement has previously been attributed to nonhomologous end joining (NHEJ), as the limited homology is not sufficient for the use of homology-dependent methods such as non-allelic homologous recombination (NAHR) (18). However, another mechanism, microhomology-mediated break-induced replication (MMBIR), which uses the replication machinery rather than recombination to explain this kind of rearrangement, has recently been proposed (19).

In short, the MMBIR model proposes that a single doublestranded break (DSB) resulting from a broken replication fork can cause a template switch in which the 3'-end anneals to another open replication fork. For this switch to occur, the two replication forks need only to share a few bases of microhomology and be located close to each other in the replicating nucleus. Depending on the orientation and chromosomal location of the new replication fork, the result can be an inversion, deletion, duplication, translocation or amplification. It is also feasible that several successive switches might occur, thus creating a more complex rearrangement (19). RAD51, a mediator of homologous recombination (HR), shows decreased expression in hypoxic tumor cells, which has been interpreted as a stress-induced switch from high fidelity HR to the more error-prone NHEJ pathway (20). However, as MMBIR has also been found to be RAD51-independent, it represents an alternative explanation in hypoxic cells, especially in the case of single DSBs where NHEJ is not an option (19).

The simple rearrangement seen in three of our samples can be readily explained by a single round of MMBIR skipping to a position upstream on the same chromosome, thus creating a rolling circle that can be further amplified (Fig. 2B). In the slightly more complicated case, a MMBIR model with two successive template switches could explain the complexity seen at the junction, first creating a small inversion and then a rolling circle (Fig. 2C). It has been proposed that features affecting the secondary structure of the DNA could locally lead to stalled replication forks and an increased genomic instability $(19,21)$. A fragile site (FRA2Ctel) located near the $M Y C N$ amplicon breakpoints has recently been identified. This fragile site was reported to be AT-rich and with significantly higher flexibility peaks than non-fragile regions of the genome, suggesting that secondary structures could form in this region (17). Although it remains unclear how far into the surrounding DNA this effect will reach, it is an intriguing feature that might help to explain the non-recurrent but clustered appearance of the breakpoint distribution.

The corresponding explanation using the NHEJ mechanism would require two separate DSBs in the simpler cases, and three or four in the more complex case. It also implies that the amplified region would be excised from its native location as a part of the amplification process, thus causing a deletion at $2 \mathrm{p} 24$. In the four cases analyzed here, no PCR fragment corresponding to a deleted gene region could be detected in the genome of the tumors, indicating that the native locus has not been excised during the amplification process. However, an event that initially created a larger deletion or translocation that was not detectable with our PCR system cannot be excluded. In the case of NBL08, a deletion much larger than the amplified area was indicated from the SNP-array data, although it could not be found using our PCR approach. This probably reflects a more complex scenario than expected for this case, for example a translocation event, which is undetectable using SNP arrays.

To our knowledge, this is the first finding of a rolling-circle mechanism caused by MMBIR in a tumor. Although all of the rearrangements presented in this article represent simple MMBIR events with only one or two template switches, 
A

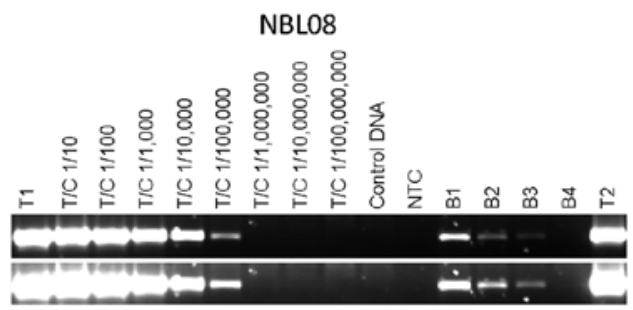

B

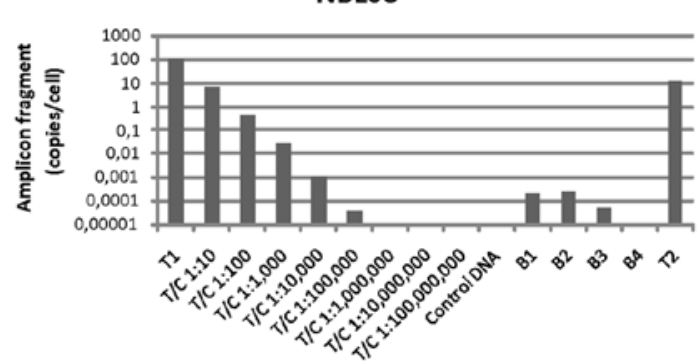

NBL11

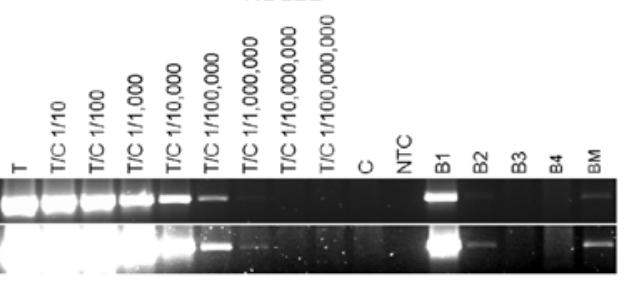

NBL11

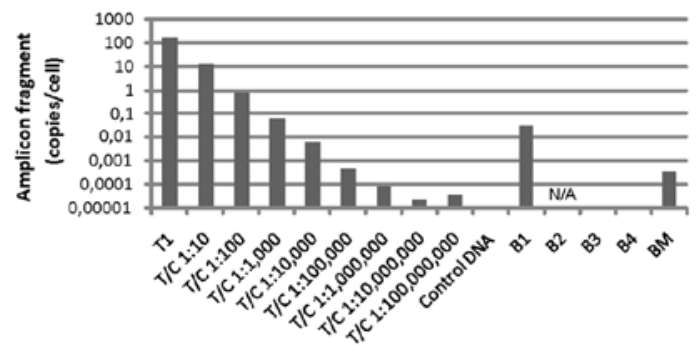

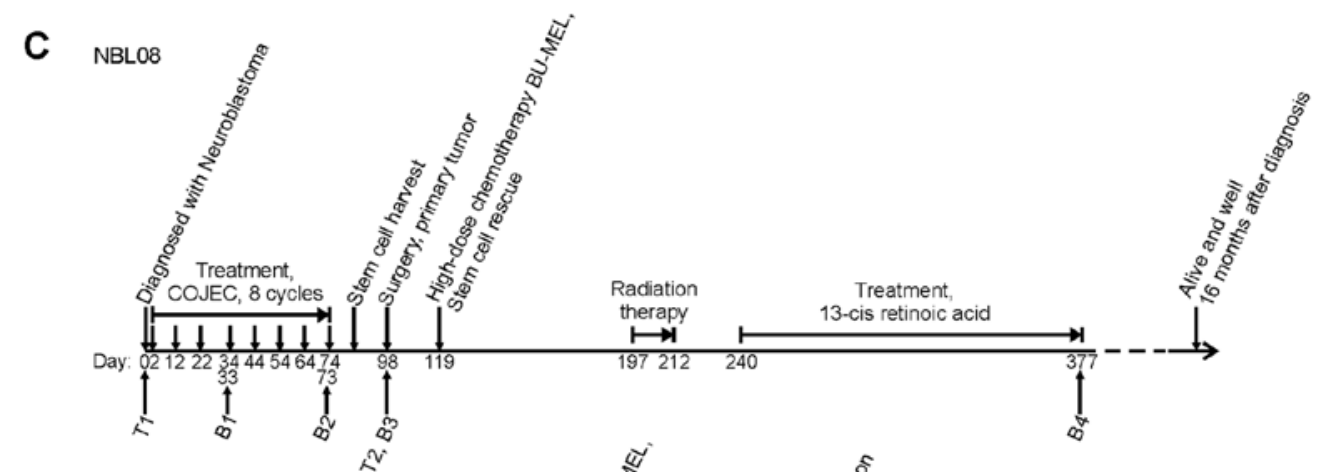

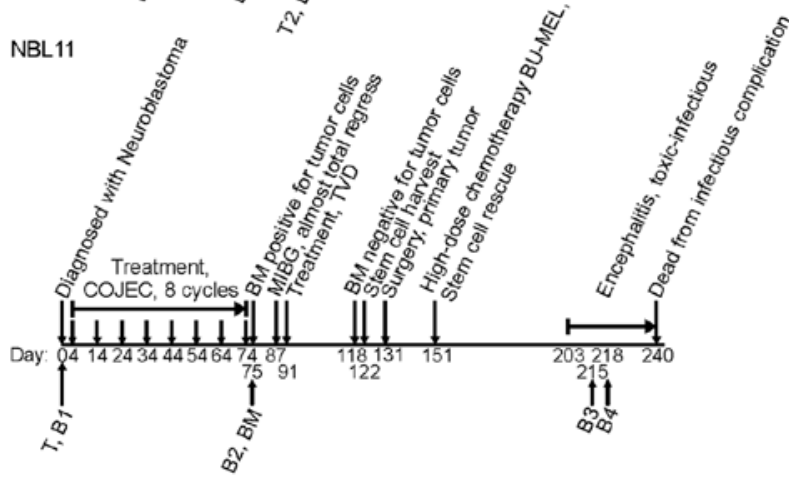

Figure 4. MRD detection using a semi-quantitative or quantitative PCR assay. (A) The semi-quantitative PCR assay is capable of detecting tumor DNA down to $1 / 10^{5}$ in NBL08 and $1 / 10^{6}$ in NBL11 in serial dilutions of tumor/control DNA. Tumor-specific DNA was detected in the first three blood samples and the second tumor sample from NBL08 as well as the two first blood samples and the bone marrow sample from NBL11. In order to clearly visualize also the fragments of low intensity, an additional brighter picture is also provided for each gel. (B) QPCR results given as the copies of amplicon junction/cell, normalized against an endogenous control fragment at chromosome 20. Detection limits are similar to the semi-quantitative PCR assay. Tumor-specific DNA was detected in the first three blood samples from NBL08 as well as the first blood sample and the bone marrow sample from NBL11. B2 from NBL11 was excluded due to lack of template. (C) Timelines representing the clinical progression for patients NBL08 and NBL11. Important events, such as treatments, disease progression and other analyses of interest are indicated as well as the time when the research samples were taken. Time is reported as days after diagnosis. [T, tumor; C, control; NTC, non-template control; B, blood; BM, bone marrow; COJEC, 8 cycle chemotherapy protocol with vincristine, carboplatin and etoposide (cycle 1 and 5), vincristine and cisplatin (cycle 2, 4, 6 and 8) and vincristine, cyclophosphamide and etoposide (cycle 3 and 7); TVD, topotecan, vincristine, doxorubicin; BU-MEL, busulphan-melphalan).

more complicated cases of MYCN amplicons presenting with multiple areas of amplification are readily found in our NB material. A detailed analysis of these amplicons will reveal whether these junctions are the result of a more complex MMBIR event.
The MYCN amplicon junction is a patient/tumor-specific target that can be used for MRD detection. Sensitive MRD detection makes it possible not only to identify patients with relapse, but also to monitor the patients throughout the treatment, thus enabling early identification of the non-responders 
that would need alternative therapies. Using QPCR with a panel of 5 different mRNA transcripts, Stutterheim et al recently showed that high risk neuroblastoma patients with negative MRD at the time of mid-induction had a significantly better outcome than those with measurable disease at this timepoint. Those with detectable MRD also at the end of induction therapy had an even worse prognosis (11). This highlights the need for several sequential samples to be analyzed during treatment and follow-up, and requires a quick, robust and sensitive method of detection. However, RNA is sensitive to degradation and variation in handling between different laboratories might lead to inconsistent results (22). Combining multiple markers also increases the amount of good quality RNA needed for the analysis. Furthermore, the mRNA expression of certain genes may change over time, for example in response to treatment, thus giving rise to false negative results.

In leukemia and lymphoma, tumor specific junctions in the form of fusion genes are routinely used for PCR-based analysis of MRD (14). Although most commonly detected at the transcript level, MRD detection using DNA as template has also been described (23). Recurrent translocations are absent from many solid tumors, including neuroblastoma, but amplifications and translocations with non-recurrent breakpoints are readily detected. Here we have shown that high density SNP arrays in combination with multiplex PCR can be used to identify tumor-specific junctions in neuroblastoma. Both the semi-quantitative and quantitative PCR were found to be sensitive enough to detect small amounts of tumor DNA $\left(1 / 10^{5}\right.$ to $1 / 10^{6}$, tumor/control) as estimated by the serial dilutions. The sensitivity could probably also be improved somewhat by using a nested PCR approach, as suggested by Bartley et al (23). Since DNA is used as template instead of mRNA it is also robust against degradation.

Furthermore, the tumor specificity of the assay renders it robust against contamination from other cell types, and whole blood or bone marrow can be used instead of serum. This enables detection of DNA contained either within circulating tumor cells (CTCs) or circulating free in the blood plasma. As whole blood has been used as the template in our assay, it is impossible to determine the origin of the detected tumor DNA. The bone marrow sample from patient NBL11, however was also found to be positive using standard cytological analyses, suggesting that at least in this sample the detected tumor DNA originated from the infiltrating tumor cells. Although the role and origin of circulating free DNA remain somewhat unclear (24), several publications report that the amount of tumor DNA in the blood reflects the size of the tumor and/or the risk of relapse in several types of cancer such as breast cancer $(24,25)$, colon cancer (26) and neuroblastoma (27,28). Some authors also stress the prognostic importance of CTCs and bone marrow micrometastasis in NB $(27,29)$. Consequently, regardless of whether the tumor DNA originates from CTCs or cell-free DNA, the persistent existence of tumor DNA in the blood or bone marrow most likely is indicative of the prognosis of the patient.

The tumor- and patient-specificity of this method is both an advantage and a limitation. As new primers have to be designed for each patient, it could be argued that this method is not sui-table as a screening method and that methods involving genome analysis with higher resolution, e.g., next generation sequencing could be used instead to define the breakpoints as exactly as possible. However, since high density arrays are increasingly used as a diagnostic tool for NB, the prerequisite for this analysis is in many cases already fulfilled making a tailormade patient-specific assay possible. Furthermore, running multiplex PCR reactions with primers carrying universal tags makes it possible to test many primers simultaneously and to sequence the junction directly from the multiplexed reaction. Once the primers are validated and a tumor-specific fragment has been achieved, numerous follow-up samples can readily be analyzed using a simple PCR reaction. It is also important to stress that time is crucial when producing tools for clinical follow-up of cancer patients. With the method devised here we have a reasonably priced tumor/patient-specific PCR assay ready, within 2-3 weeks after tumor material has arrived at the DNA laboratory. The next-generation sequencing strategies are presently not that fast, and the cost of analysis is considerably higher. It is likely, however that the methods that involve high throughput sequencing, which are now research tools in the analysis of primary tumors, will eventually be used in the clinical diagnosis of neuroblastoma tumors.

In summary, we present a detailed analysis of the novel junctions in a subset of $M Y C N$ amplifications and propose that the microhomology found at these junctions indicates a rolling-circle amplification caused by MMBIR, which to our knowledge is the first such finding in tumor material. Furthermore, we have shown that SNP-arrays, commonly used in neuroblastoma diagnostics, is a cost-efficient way to identify tumor specific junctions that are suitable targets for MRD detection, and that tumor-specific DNA can be readily detected and quantified in either blood or bone marrow from patients with $M Y C N$ amplification.

\section{Acknowledgements}

This study was supported by the Swedish Cancer Society (09/1217 TM), the Swedish Children's Cancer Foundation (07/098 TM) and the Assar Gabrielsson foundation (FB 08-86 $\mathrm{HK})$. We are grateful for help and access to instrumentation provided by the Sahlgrenska Academy Genomics Core Facility.

\section{References}

1. Maris JM and Matthay KK: Molecular biology of neuroblastoma. J Clin Oncol 17: 2264-2279, 1999.

2. Caren H, Erichsen J, Olsson L, et al: High-resolution array copy number analyses for detection of deletion, gain, amplification and copy-neutral $\mathrm{LOH}$ in primary neuroblastoma tumors: four cases of homozygous deletions of the CDKN2A gene. BMC Genomics 9: 353, 2008.

3. Michels E, Vandesompele J, Hoebeeck J, et al: Genome wide measurement of DNA copy number changes in neuroblastoma: dissecting amplicons and mapping losses, gains and breakpoints. Cytogenet Genome Res 115: 273-282, 2006.

4. Scott D, Elsden J, Pearson A and Lunec J: Genes co-amplified with MYCN in neuroblastoma: silent passengers or co-determinants of phenotype? Cancer Lett 197: 81-86, 2003.

5. Brodeur GM: Neuroblastoma: biological insights into a clinical enigma. Nat Rev Cancer 3: 203-216, 2003.

6. Corvi R, Amler LC, Savelyeva L, Gehring M and Schwab M: MYCN is retained in single copy at chromosome 2 band p23-24 during amplification in human neuroblastoma cells. Proc Natl Acad Sci USA 91: 5523-5527, 1994. 
7. Stark GR, Debatisse M, Giulotto E and Wahl GM: Recent progress in understanding mechanisms of mammalian DNA amplification. Cell 57: 901-908, 1989.

8. Amler LC and Schwab M: Amplified N-myc in human neuroblastoma cells is often arranged as clustered tandem repeats of differently recombined DNA. Mol Cell Biol 9: 4903-4913, 1989.

9. Hunt JD, Valentine $\mathrm{M}$ and Tereba A: Excision of N-myc from chromosome 2 in human neuroblastoma cells containing amplified $\mathrm{N}$-myc sequences. Mol Cell Biol 10: 823-829, 1990.

10. Schwab M, Alitalo K, Klempnauer KH, et al: Amplified DNA with limited homology to myc cellular oncogene is shared by human neuroblastoma cell lines and a neuroblastoma tumour. Nature 305: 245-248, 1983.

11. Stutterheim J, Zappeij-Kannegieter L, Versteeg R, Caron HN, van der Schoot $\mathrm{CE}$ and Tytgat GA: The prognostic value of fast molecular response of marrow disease in patients aged over 1year with stage 4 neuroblastoma. Eur J Cancer 47: 1193-1202, 2011.

12. Stutterheim J, Gerritsen A,Zappeij-Kannegieter L, et al: Detecting minimal residual disease in neuroblastoma: the superiority of a panel of real-time quantitative PCR markers. Clin Chem 55: 1316-1326, 2009.

13. Cheung IY, Feng Y, Gerald W and Cheung NK: Exploiting gene expression profiling to identify novel minimal residual disease markers of neuroblastoma. Clin Cancer Res 14: 7020-7027, 2008

14. Van der Velden VH, Hochhaus A, Cazzaniga G, Szczepanski T, Gabert $\mathbf{J}$ and van Dongen JJ: Detection of minimal residual disease in hematologic malignancies by real-time quantitative PCR: principles, approaches, and laboratory aspects. Leukemia 17: 1013-1034, 2003.

15. McBride DJ, Orpana AK, Sotiriou C, et al: Use of cancer-specific genomic rearrangements to quantify disease burden in plasma from patients with solid tumors. Genes Chromosomes Cancer 49: 1062-1069, 2010.

16. Leary RJ, Kinde I, Diehl F, et al: Development of personalized tumor biomarkers using massively parallel sequencing. Sci Trans Med 2: 20ra14, 2010.

17. Blumrich A, Zapatka M, Brueckner LM, Zheglo D, Schwab M and Savelyeva L: The FRA2C common fragile site maps to the borders of MYCN amplicons in neuroblastoma and is associated with gross chromosomal rearrangements in different cancers. Hum Mol Genet 20: 1488-1501, 2011.
18. Hastings PJ, Lupski JR, Rosenberg SM and Ira G: Mechanisms of change in gene copy number. Nat Rev Genet 10: 551-564, 2009.

19. Hastings PJ, Ira G and Lupski JR: A microhomology-mediated break-induced replication model for the origin of human copy number variation. PLoS Genet 5: e1000327, 2009.

20. Bindra RS, Schaffer PJ, Meng A, et al: Down-regulation of Rad51 and decreased homologous recombination in hypoxic cancer cells. Mol Cell Biol 24: 8504-8518, 2004

21. Bacolla A, Wojciechowska M, Kosmider B, Larson JE and Wells RD: The involvement of non-B DNA structures in gross chromosomal rearrangements. DNA Repair (Amst) 5: 1161-1170, 2006.

22. Beiske K, Burchill SA, Cheung IY, et al: Consensus criteria for sensitive detection of minimal neuroblastoma cells in bone marrow, blood and stem cell preparations by immunocytology and QRT-PCR: recommendations by the International Neuroblastoma Risk Group Task Force. Br J Cancer 100: 1627-1637, 2009.

23. Bartley PA, Ross DM, Latham S, et al: Sensitive detection and quantification of minimal residual disease in chronic myeloid leukaemia using nested quantitative PCR for BCR-ABL DNA. Int J Lab Hematol 32: e222-228, 2010.

24. Fleischhacker $M$ and Schmidt B: Circulating nucleic acids (CNAs) and cancer - a survey. Biochim Biophys Acta 1775: 181-232, 2007.

25. Kohler C, Radpour R, Barekati Z, et al: Levels of plasma circulating cell free nuclear and mitochondrial DNA as potential biomarkers for breast tumors. Mol Cancer 8: 105, 2009.

26. Diehl F, Schmidt K, Choti MA, et al: Circulating mutant DNA to assess tumor dynamics. Nat Med 14: 985-990, 2008.

27. Fukuda M, Miyajima Y, Miyashita Y and Horibe K: Disease outcome may be predicted by molecular detection of minimal residual disease in bone marrow in advanced neuroblastoma: a pilot study. J Pediatr Hematol Oncol 23: 10-13, 2001.

28. Gotoh T, Hosoi H, Iehara T, et al: Prediction of MYCN amplification in neuroblastoma using serum DNA and real-time quantitative polymerase chain reaction. J Clin Oncol 23: 5205-5210, 2005.

29. Kuroda T, Morikawa N, Matsuoka K, et al: Prognostic significance of circulating tumor cells and bone marrow micrometastasis in advanced neuroblastoma. J Pediatr Surg 43: 2182-2185, 2008. 